Classification

Physics Abstracts

$42.30-06.30 . \mathrm{C}-06.30 . \mathrm{M}-06.60-07.80-81.40 . \mathrm{L}$

\title{
The Use of Morphological Filters in Computing Displacement Fields in a Sequence of S.E.M. Images
}

\author{
Najet Ben Amar, Azeddine Beghdadi and Patrick Viaris de Lesegno \\ Laboratoire des Propriétés Mécaniques et Thermodynamiques des Matériaux, CNRS, \\ Institut Galilée, Université Paris Nord, avenue J.B. Clément, 93430 Villetaneuse, France
}

(Received May 15; accepted November 12, 1996)

\begin{abstract}
In order to quantify the material deformation when submitted to mechanical solicitations, we propose a new technique based on a set of image analysis operations. It consists in measuring the displacements of some geometrical points selected on the surface of the deformed material and considered as landmarks. More details about this technique, presented below, are justified by actual applications.
\end{abstract}

\section{Introduction}

The optimisation of material properties requires the control of both their surface morphology and their mechanical behaviour in front of some external solicitations. Up to now and because we cannot extract the inner information of the studied material we content ourself with the information on the surface of this material. The surface is analysed by the way of a Scanning Electron Microscope (SEM). This later, combined with a microelectrolitographic technique, gives us the opportunity to follow with a precision, about $0.1 \mu \mathrm{m}$, the evolution of a material deformation. In fact, the microelectrolitographic technique consists in depositing a metallic layer'(generally a gold layer) forming a grid on the surface of the studied material. The grids may be composed of lines or dots. These laters are difficult to follow in high rate deformations because their shape can drastically change between two steps. Besides, they can be confused with some artefacts present in the image. This is the reason why we deal only with line grids instead of dots. In addition, the experiments show that line grids deliver a better pattern of crystallographic slips, cracks or decohesions.

There are several methods, based on image analysis techniques, which allow to obtain accurate measurements of local displacements fields [1-4]. Among them, many are based on the analysis of the cross correlation function. They maximise the correlation factor in order to detect similar zones between two images or between some predefined models and one image. With these techniques, only low deformation material rates can be considered, because they require the aspect of microgrid intersections to remain the same between two deformation states. This condition is 
not usually verified. Besides, this kind of methods is noise sensitive and much time consuming since they compute the correlation product over all the tested domain. Other methods based on some morphological operations exist [3]. They are limited to low deformation rates because they suppose the polygons of the microgrid to be regular over all the mechanical solicitation.

In this paper we propose a new technique based on some particular shapes recognition principle using morphological operations. The first part of this paper deals with a description of processed images and the useful information that they contain. In the second part, we describe the new method which consists of a set of image treatment tools. This part is followed by experimental results obtained on actual images. The last section is devoted to the main conclusions and opened problems.

\section{Description of Studied Images}

As previously said, the studied images come from a SEM. They represent a surface state of the sample on which is deposited a gold microgrid by a microelectrolitographic technique [5]. The microgrids are obtained by sweeping the electronic beam of the scanning microscope. They have a step of a few micrometers, while the bars thickness is equal to $1 / 10$ of grid step. A typical value of $0.3 \mu \mathrm{m}$ is often used, but $0.15 \mu \mathrm{m}$ may be obtained. The scanned images are digitised according to a raster of $768 \times 576$ or $1536 \times 1158$ pixels and quantized to 256 grey levels. The deformation of the grid expresses the surface state of the material. Thus, it gives indications on the local scale mechanisms that occur during the deformation of the sample. Figure 1 shows the studied material observed at two different deformation states: Figure 1a represents a low rate. It could be noticed that the microgrid is quite regular over all the image, whereas Figure 1b shows a high deformed sample in which the polygons of the grid have not the same shape.

\section{Description of the Proposed Method}

The proposed method operates in a complete automatic mode and consists of two steps: the first one deals with the localisation of some geometric landmarks of the deposited microgrid, whereas the second one allows the estimation of all landmark displacements by comparing two images corresponding to two different deformation rates. More details about each step are given in the following.

3.1 Recognition Step of Geometric Landmarks. - It corresponds to determine all microgrid line intersections, namely nodes. For this, and in order to facilitate this task, we proceed firstly by filtering the image to eliminate all artefacts and to enhance the relevant information. Secondly, we extract the microgrid lines in order to facilitate the nodes detection. Microgrid landmarks, as they were chosen, correspond to the intersections of columns and rows, the definition of all possible shapes of the different crossings is necessary. Finally, we recognise all these defined shapes, in the image under study, by some binary morphological operations. The set of these operations is described below.

3.1.1 Filtering Operation. - Images coming from a SEM are often noisy and necessitate some filtering operations. We apply a cross median filter followed by a morphological dilation and closing. The median filter, in contrast with some linear filters [6-8], reduces the noise without sensibly delocalizing the contour lines. For this application, it preserves the linearity and the morphology of microgrid lines. Whereas, the grey level morphological operations allow, on the one hand, to enhance the information contained in the microgrid lines by a grey level dilation and, on the other hand, to connect some fragmented grid segments by a closing. Besides, the dilation 
a)

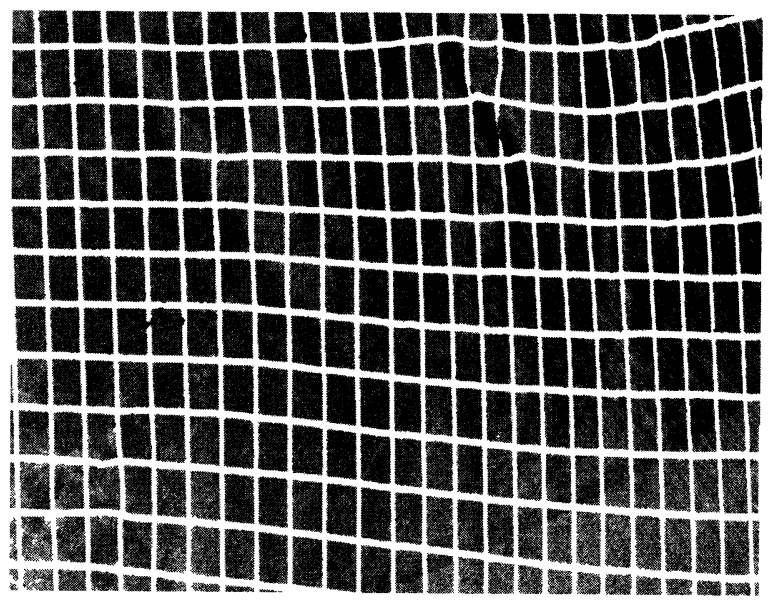

b)

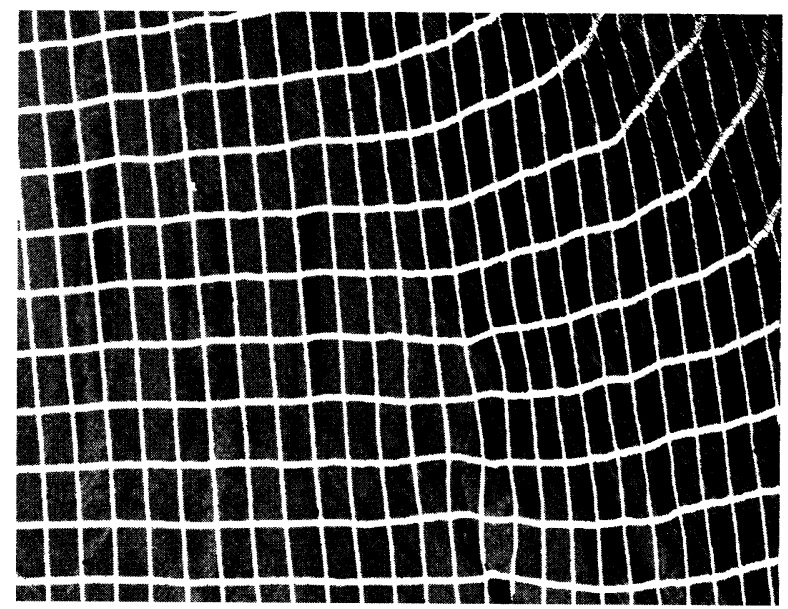

Fig. 1. - Original images corresponding to two different deformation states: a) low deformed material; b) high deformed material.

allows to obtain thicker grid lines in order to protect them from some additional post-treatments. All these filtering operations are performed with $3 \times 3$ structuring elements. Indeed, the use of large sized structuring element is much time consuming for an automatic analysis and does not preserve the initial structure of microgrid crossings as proved in [9].

3.1.2 Thresholding Operation. - To isolate the microgrid lines from the background, a local thresholding operation is applied. The pre-processed image is subdivided into regions of similar sizes. Then a local grey-level thresholding is performed in each region. The optimal threshold corresponds to the grey level which maximises the inter-class variance [10,11]. The difficulties of a local thresholding operation are the choice of the shape and the size of each region. The later must be, in one hand, very large in order to represent the entire image and, in the other hand, sufficiently small in order to consider all the luminance variations of the image. In our case we use non overlapping square regions with a raster of 64 per 64 pixels. As shown in Figure 2, which corresponds to the thresholded image of Figure 1a, only grid lines are preserved in the thresholded image. 


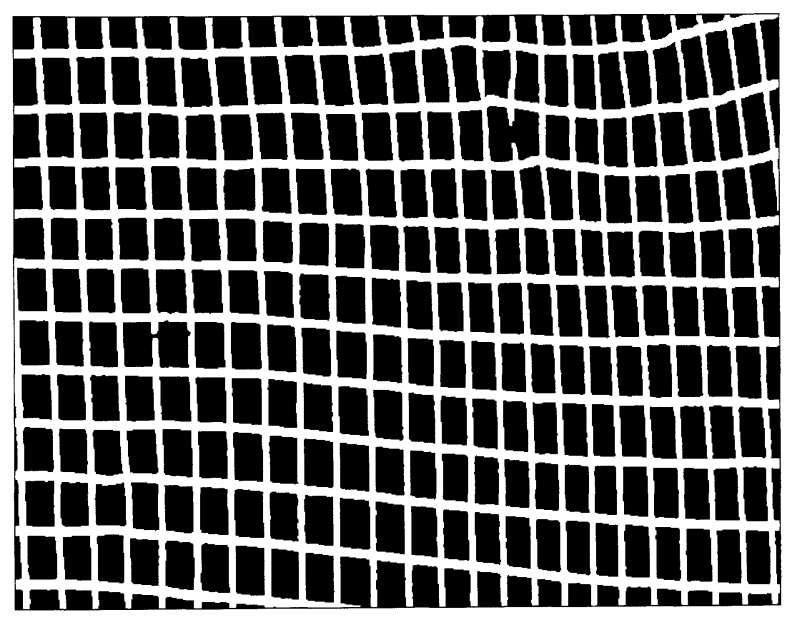

Fig. 2. - Thresholded image.

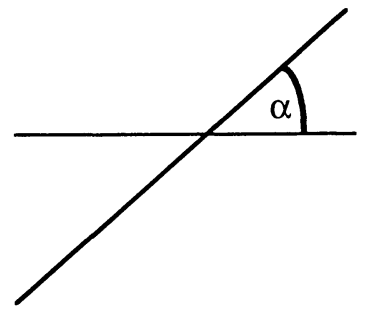

Fig. 3. - Geometric representation of one cross.

3.1.3 Different Crossing Shapes Definition. - As shown in all precedent figures, one can notice that the microgrid is generally composed by horizontal and vertical bars. The intersections of these lines correspond to the searched nodes. So, the node localisation becomes possible by determining all these intersections and by defining the different models corresponding to the different shapes of these crossings. A simple visual analysis leads to select crosses as models. Each cross is defined by an angle $\alpha$ (see Fig. 3).

In order to accelerate the search procedure of theses crosses, we chose the values of $\alpha_{i}$ such as:

$$
\alpha_{i}=k \pi / 4 ; \quad k=1,2,3 .
$$

Consequently, six cross models could be defined as shown in Figure 4.

It could be noticed that the adaptation of the cross segments to the microgrid line thickness is necessary. For this, horizontal and vertical line thicknesses, namely respectively $C_{1}$ and $C_{2}$, are determined and the cross segments are defined as shown, for example for the (a) model, in Figure 5.

3.1.4 Nodes Recognition. - Mathematical morphology [12-14] studies the shape of objects using some set operations such as inclusion, intersection, complementation, etc. The image is considered as a set of shapes that we try to recognise by using some predefined prototype named structuring element. The recognition operation consists in extracting all image points which gives positive responses to the total inclusion of the prototype in the analysed structures. In the studied 


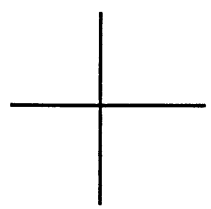

(a)

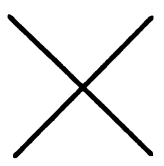

(d)

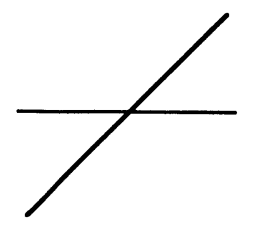

(b)

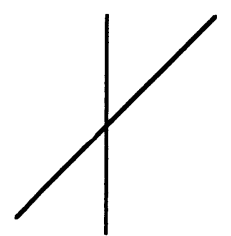

(e)

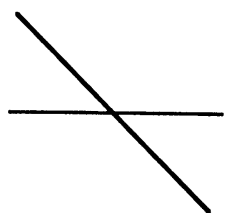

(c)

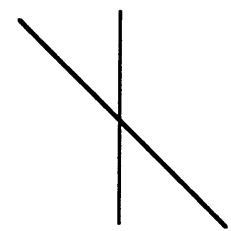

(f)

Fig. 4. - Different models to be recognised.

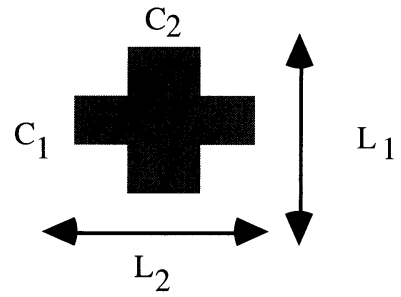

(a)

Fig. 5. - Definition of the cross of (a) model, where $L_{1}=3 C_{2}$ and $L_{2}=3 C_{1}$.

problem, the localisation of lines intersections (nodes) becomes easier, once all models are defined, by binary morphological erosions. The structuring elements correspond to the six defined crosses. We test the inclusion of these crosses in the intersections and we search all image locations which are able to contain, at least one of the six structuring elements. The operation is an union of six erosions and the inclusion test is made by using the following algorithm.

For all grid points Do $/ *$ grid point $=$ white pixel $* /$

(1) Erosions with the six defined crosses

If only one cross is included in the line intersections

Then one node is detected

Else

I* more than one response */

Increase the crosses dimensions

Got to (1)

End If

End For 
Problem. - The inclusion test of the six crosses gives satisfactory results with regards to the localisation quality of the microgrid intersections for all studied images. Nevertheless, this morphological operation is very slow and seems to be non adequate for an automatic process. We remind that one cross, according to the definition of Figure 5, necessitates $5 C_{1} C_{2}$ tests per microgrid pixel. Besides, the studied images have a size of $768 \times 576$ or $1536 \times 1158$ pixels, so that, they require a large amount of time to search all microgrid crossings. In order to increase the speed of the computation of this morphological operation, some improvements are possible $[15,16]$. For the present study, the search of all nodes is done on binary images, so that we can only use the cross edges instead of the entire crosses. For this, we consider that microgrid lines don't contain holes (black pixels) due to the pre-treatments, as dilation and closing grey level operations. Under these conditions, a binary erosion by a structuring element $B$, with any shape, gives similar results as the same operation restricted to the edge, $\delta B$, of this element:

$$
B^{B}(X) \Leftrightarrow E^{\delta B}(X)
$$

This similarity is justified by the following property [17]:

$$
\text { if } B_{1} \subset B_{2} \text { then } E^{B_{2}}(X) \subset E^{B_{1}}(X)
$$

where $B_{1}$ and $B_{2}$ are two different structuring elements.

It is well known, that an edge of any shape is included in this shape and so that this similarity is verified for our problem. Thus, the number of tests per pixel is considerably reduced passing from $5 C_{1} C_{2}$ operations to only $6\left(C_{1}+C_{2}\right)$ which corresponds to one cross perimeter. Furthermore, the obtained results with this second solution are quite similar to those obtained with the first one.

Applications. - The original image is the result of a succession of many operations: median filter, morphological dilation and closing, and a local grey-level thresholding adapted from the algorithm of Reddi et al. [11]. From this image, we determine the microgrid rows and columns thickness using some operations based on local gradient computations and contours tracking. Then, we adapt the six defined cross models, as described previously. Finally, we apply the previous algorithm to node detections. As shown in Figure 6, one can see that all nodes are detected. This detection necessitates 1 minute on a RISC processor whereas a semi-automatical method [9] takes two hours. We notice that over the tested images the six defined cross models of Figure 4 are fully satisfactory. They are sufficient to detect all microgrid nodes in slightly deformed material images. It is well known that the definition of other models will give a more accurate localisation but it will be a more time consuming.

The accuracy of the detection is greatly influenced by the quality of the microgrid. When this later remains regular in thickness, all over the deformation action, the node localisation is done with a rate of $100 \%$. Otherwise, this rate decreases and the non detected nodes are due to some irregularities in the microgrid morphology.

3.2 Deformation Estimation. - To compute, with accuracy, local strain field components, it is necessary to have at least two images corresponding to different state of deformation. We begin by detecting the coordinates of all microgrid nodes of each image. We apply, for this, the algorithm given previously. Then, we determine the components of each strain field vector by measuring the displacement of each node as follows:

$$
\mathbf{d}^{(k)}=\left(\begin{array}{c}
X_{2}^{(k)}-X_{1}^{(k)} \\
Y_{2}^{(k)}-Y_{1}^{(k)}
\end{array}\right)
$$




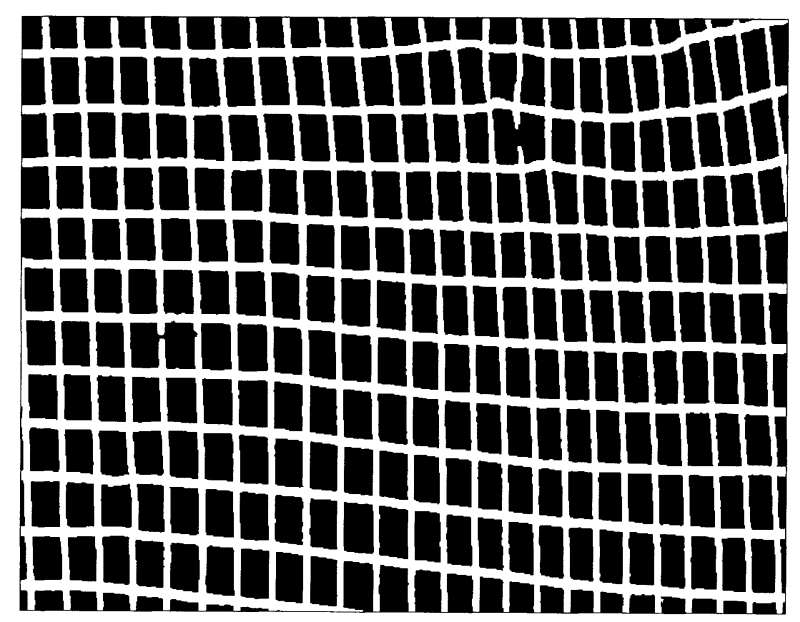

Fig. 6. - Nodes detection of Figure 1a.

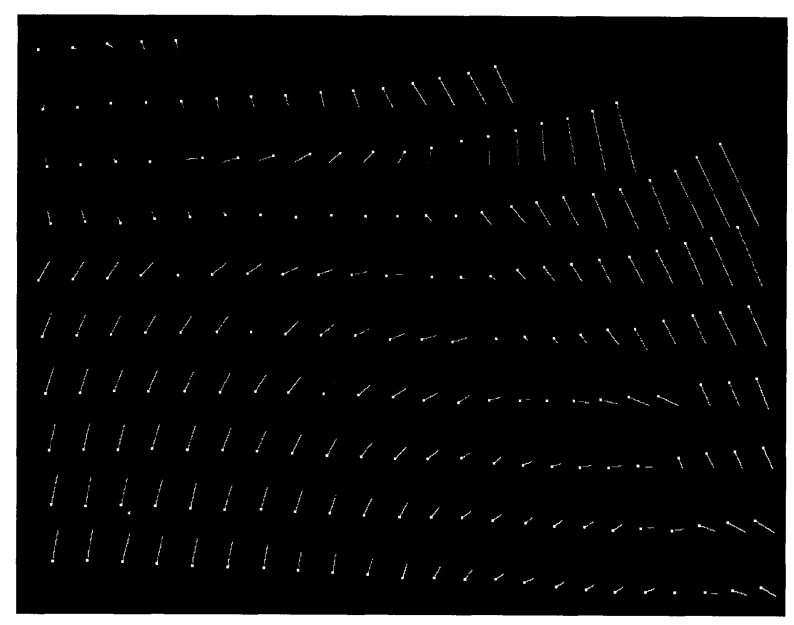

Fig. 7. - Strain field vectors representation.

where $\mathbf{d}$ corresponds to the strain field vector. $\left(X_{1}^{(k)}, Y_{1}^{(k)}\right)$ and $\left(X_{2}^{(k)}, Y_{2}^{(k)}\right)$ represent the coordinate of the $k$ th node in two successively deformed images $\mathrm{I}_{1}$ and $\mathrm{I}_{2}$.

The direction of this vector is chosen such as the origin point, $\left(X_{1}^{(0)}, Y_{1}^{(0)}\right)$, is in the initial image and the arrival point, $\left(X_{2}^{(0)}, Y_{2}^{(0)}\right)$, is in the second image. The representation of node displacements of the image given in Figure $1 \mathrm{~b}$ with respect to Figure 1a, is expressed by Figure 7 . All over the tested images, the deformation error is about the pixel, due, on the one hand, to the irregularities in the microgrid, as said previously, and on the other hand to the errors of matching which can be accumulated.

One can notice that these vectors express both the main direction of the sample deformation and also the evolution of this deformation in each microgrid landmark and all over the plane of the treated material. It is possible to evaluate the $3 \mathrm{D}$ deformation, by using two SEM surface images, instead of one, for each deformation state: from one reference image and a $30^{\circ}$ tilted image, 
one can localise, using the proposed method, all corresponding landmarks in the two images; the Z-components are then deduced and the 3D surface strain field vectors are determined. It is then possible to compute the six components of deformation gradient tensor $(\mathbf{F})$ instead of only four and without using a 3D information [18].

\section{Conclusions}

In this paper, an automatic and non adaptive technique is presented. It allows to quantify the evolution of the material surface states.

This technique shows that an entire automatic analysis on material images is possible. This analysis is decomposed into two steps: landmarks localisation and displacement field vectors estimation. The results indicate that special morphological erosions (with adapted structuring element shapes) on thresholded images allow to localise, with accuracy, the microgrid crossings. Displacement vectors are determined by simultaneously analysing two images and by computing the displacement of each node between the two images. We notice that the node localisation is totally achieved when the microgrid is slightly damaged, otherwise the nodes are partially detected and the six crossing models are insufficient. Additional models could improve the result but at the expense of time computing. Other solutions are under study [19].

\section{References}

[1] Allais L., Bornert M., Bretheau T. and Caldemaison D., Strain in a heterogeneous elastoplastic material, Acta Metall. Mater. 42 (1994) 3865-3880.

[2] Ben Amar N., Beghdadi A. and Viaris de Lesegno P., An all-digital method for accurate measurement of mechanical deformations, Scanning J. Microsc. 18 (1996) 327-330.

[3] Tuzikov A., Soille P. and Jeulin D., Extraction of grid patterns on stamped metal sheets using mathematical morphology, Actes 11 Conférence IAPR La Hague, 30 août-3 septembre 1992.

[4] Jähne B., Image Processing Concepts, Algorithms and Scientific Applications (Springer Verlag, Second Edition, 1993), pp. 122-154.

[5] Makie S. and Beaumont S.P., Materials and processes for nanometer lithography, Solid State Technol. (1985) 117-122.

[6] Conrad Bovik A. and Munson D.C., Edge detection using median comparisons", Comput. Vision, Graphics and Image Process. 33 (1986) 377-389.

[7] Huang T.S., Yang G.J. and Tang G.Y., A fast two dimentional median filtering algorithm, IEEE Trans. Acoust., Speech Signal Process. ASSP-27 (1979) 13-18.

[8] Coyle E.J., Lin J.H. and Gabbouj M., Optimal stack filtering and the estimation of structural approaches to image processing, IEEE Trans. Acoust., Speech Signal Process. 37 (1989) 2037-2066.

[9] Ben Amar N., Développement d'Outils de Traitement d'Images Numériques pour le Suivi et l'Évaluation de la Déformation Plastique des Matériaux, Thèse de Doctorat de l'Université Paris Nord (1995).

[10] Otsu N., A Threshold selection Method from Gray Level Histograms, IEEE Trans. Syst. Man Cyber. 1 (1979) 62-66.

[11] Reddi S.S., Rundin S.F. and Keshavan H.R., An Optimal Multiple Threshold Scheme for Image Segmentation, IEEE Trans. Syst. Man Cyber. SMC-14 (1984) 661-665.

[12] Coster M. and Chermant J.L., Précis d'analyse d'images ( $2^{\mathrm{e}}$ édition, Éditions CNRS, Paris, 1989). 
[13] Serra J., Image Analysis and Mathematical Morphology (London, Academic Press, 1982).

[14] Serra J., Image Analysis and Mathematiçal Morphology, part II: Theoretical advances (Academic Press, 1988).

[15] Crabtree S.J., Yuan L.P. and Ehrlich R., A fast accurate erosion-dilation method suitable for microcomputer, Comput. Vision Graphics and Image Process. 55 (1991) 283-290.

[16] Van Den Boomgaard R. and Van Balen R., Methods for fast morphological image transforms using bitmapped binary images, Comput. Vision Graphics and Image Process. 54 (1992) 252-258.

[17] Ballar D.H. and Christopher M.B., Computer Vision (Prentice-Hall, Inc., Englewood, New Jersey, 1982).

[18] Ben Amar N., Viaris De Lesegno P. and Beghdadi A., Automatic determination of material displacement fields using image analysis techniques, accepted for publication in the Proceeding of DVM Congress (ed. Woodhead Publishing Ltd, 1996).

[19] Monteil J, Beghdadi A. and Viaris De Lesegno P., The use of optical flow techniques for material deformation measurements, MICRO'96, Londres (1996): 